\title{
La tutela dei diritti umani nell'ambito dell'attività di Frontex
}

\author{
di Andrea Spagnolo*
}

\begin{abstract}
Sommario: 1. Premessa e piano d'indagine - 2. Segue. Alcune considerazioni generali sulle norme in materia di diritti umani applicabili nel controllo alle frontiere esterne dell'UE - 3. L'ambito di applicazione delle norme convenzionali in materia di diritti umani. In particolare, l'applicazione extraterritoriale dell'obbligo di non respingimento - 4. Il contenuto dell'obbligo di non respingimento così come recepito nel regolamento n. 656/2014 - 5. Il ruolo di Frontex nelle operazioni di ricerca e salvataggio in mare: il dibattito in merito alla configurabilità di un "diritto a essere salvati" - 6. La responsabilità per violazioni dei diritti umani nell'ambito delle attività di Frontex - 7. L'effettività dei diritti umani nell'ambito delle operazioni Frontex: un problema aperto - 8. Segue. Il monitoraggio dei diritti umani nell'ambito di Frontex: una possibile conclusione
\end{abstract}

\section{Premessa e piano d'indagine}

L'attività dell'Agenzia europea per la gestione della cooperazione operativa alle frontiere esterne (Frontex) ${ }^{1}$ è da diverso tempo oggetto di attenzioni sotto il profilo della tutela dei diritti umani. ${ }^{2}$ È innegabile, infatti, che

\footnotetext{
*Assegnista di ricerca in Diritto internazionale, Università della Valle d'Aosta.

1. Regolamento (CE) n. 2007/2004 del Consiglio del 26.10.2004, che istituisce un'Agenzia europea per la gestione della cooperazione operativa alle frontiere esterne degli Stati membri dell'Unione europea, in GUUE L 349 del 25.11.2004, p. 1 ss. Non è qui possibile esplorare nel dettaglio la complessa attività dell'Agenzia. Per un'analisi completa, sul punto, si rimanda a A. Neal, Securization at risk at the EU border: the origins of Frontex,in Journal of Common Market Studies, 2009, p. 333 ss.; A Baldaccini, Extraterritorial Border Controls in the EU: The Role of Frontex in Operations at Sea, in Extraterritorial Immigration Control, B. Ryan, V. Mitsilegas (eds), L'Aja 2010, p. 229 ss.; G. Caggiano, Attività e prospettive d'intervento dell'Agenzia Frontex nel Mediterraneo, in Europa e Mediterraneo. Le regole per la costruzione di una società integrata. Atti del XIV Convegno della SIDI, Napoli 2010, p. 403 ss.

2. Sul punto, la dottrina è già piuttosto ampia e corposa. Si vedano, inter alia, A. FischerLescano, T. Löhr, T. Tohidipur, Border Controls at Sea: Requirements under International Human Rights and Refugee Law, in International Journal of Refugee Law, 2009, p. 256 ss.; E. Papastavridis, "Fortress Europe" and Frontex: within or without international law?, in Nordic Journal of International Law, 2010, p. 75 ss.; A. Liguori, N. Ricciuti, Frontex ed il
}

Diritto, immigrazione e cittadinanza XVI, 3-4.2014 
la stessa raison d'être delle operazioni condotte nell'ambito dell'Agenzia comporti ingerenze più o meno giustificate nel godimento dei diritti di coloro $i$ quali attraversano il Mediterraneo sperando di potersi stabilire in Europa. ${ }^{3}$

Una delle attività di Frontex maggiormente contestate è l'intercettazione di barconi di immigrati in mare e il conseguente respingimento degli stessi verso Stati terzi dove i loro diritti sono esposti al rischio di essere violati.

In relazione a quest'ultima ipotesi, si pensi alle operazioni congiunte coordinate da Frontex cui partecipano più Stati membri dell'Unione europea (UE) - e non solo - oppure agli interventi rapidi alle frontiere ${ }^{4}$ in cui sono impiegate le squadre europee di guardie di frontiera. ${ }^{5}$

L'intercettazione di migranti in mare ha già sollevato - in tempi recenti ${ }^{6}$ più di una perplessità in relazione alla violazione dei diritti di coloro i quali si trovano in siffatte condizioni. ${ }^{7}$

rispetto dei diritti umani nelle operazioni congiunte alle frontiere esterne dell'Unione europea, in Diritti umani e diritto internazionale, 2012, p. 554.

3. La prospettiva è quella, ampia, del rispetto dei diritti umani nel contrasto all'immigrazione clandestina $\mathrm{e}$, più in generale, nella gestione dei flussi migratori, soprattutto per mare. Per una ricognizione degli strumenti di diritto internazionale e dell'Unione europea adottati a tal fine si veda S. Trevisanut, Immigrazione irregolare via mare, diritto internazionale e diritto dell'Unione europea, Napoli 2012. In generale sulla gestione dei flussi migratori e il rispetto dei diritti umani cfr. G. Palmisano, Trattamento dei migranti clandestini e rispetto degli obblighi internazionali in materia di diritti umani, in Diritti umani e diritto internazionale, 2009 p. 509 ss.

4. Regolamento (CE) n. 863/2007 del Parlamento europeo e del Consiglio dell'11.7.2007, che istituisce un meccanismo per la creazione di squadre di intervento rapido alle frontiere e modifica il regolamento (CE) n. 2007/2004 del Consiglio limitatamente a tale meccanismo e disciplina i compiti e le competenze degli agenti distaccati, in GUUE L 199 del 31.7.2007, p. 30 ss.

5. Regolamento (UE) n. $1168 / 2011$ del Parlamento europeo e del Consiglio del 25.10.2011, recante modifica del regolamento (CE) n. 2007/2004 del Consiglio che istituisce un'Agenzia europea per la gestione della cooperazione operativa alle frontiere esterne degli Stati membri dell'Unione europea, in GUUE L 304 del 22.11.2011, p. 1 ss.

6. Si veda ad esempio il rapporto stilato dall'organizzazione non governativa Human Rights Watch: EU Dirty Hands. Frontex involvement in ill-treatment of migrant detainees in Greece, 21.9.2011, disponibile sul sito internet www.hrw.org.

7. Sul tema del respingimento in alto mare e la sua compatibilità con le norme poste a tutela dei diritti umani si vedano ex multis: A. Terrasi, I respingimenti in mare di migranti alla luce della Convenzione europea dei diritti umani, in Diritti umani e diritto internazionale, 2009, p. 591 ss.; E. Papastavridis, The Interception of Vessels on the High Seas, Contemporary Challenges to the Legal Order of the Oceans, Oxford 2013. 


\section{A. Spagnolo}

Si aggiunga a questa preoccupazione il tema del ruolo di Frontex nelle operazioni di ricerca e salvataggio in mare che, potenzialmente, possono essere in grado di prevenire tragedie immani. ${ }^{8}$

Non è un caso che già nel 2012 il Mediatore europeo concludeva una prima indagine sul modus operandi di Frontex, rilevando diverse criticità, proprio per ciò che concerne il rispetto dei diritti umani. ${ }^{9}$

L'indagine proprio motu del Mediatore europeo nei riguardi dell'operato di Frontex ha portato il tema del rispetto dei diritti umani nel controllo alle frontiere esterne dell'UE nell'agenda delle istituzioni europee, che sono giunte all'approvazione di un nuovo regolamento, il n. 656 del $2014,{ }^{10}$ che ha introdotto nuove norme per la sorveglianza delle frontiere marittime, in particolare per quanto riguarda la tutela dei diritti fondamentali. ${ }^{11}$

Il presente articolo si propone di fornire alcuni spunti ricostruttivi relativamente al tema della tutela dei diritti umani nell'ambito delle attività di Frontex.

L'idea di fondo è che nello spazio giuridico europeo gli obblighi in materia di diritti umani debbano essere armonizzati, soprattutto in un settore delicato come quello della tutela dei migranti. A un'armonizzazione del diritto applicabile dovrebbe seguire un'armonizzazione del regime di responsabilità dell' Agenzia e degli Stati membri che partecipano alle operazioni di Frontex, ciò che garantirebbe una tutela dei diritti efficace.

8. Il dibattito sul punto si è particolarmente acceso, soprattutto in Italia, a seguito della tragedia di Lampedusa dell'ottobre del 2013 e del successivo dispiegamento dell'operazione navale Mare Nostrum. Cfr. sul punto B. Nascimbene, L'Italia chiama, l'Europa risponde? La tragedia di Lampedusa, in Il corriere giuridico, 2013, p. 1393 ss.; Id., Lampedusa: una tragedia con molti interrogativi. Obblighi di tutela dei diritti fondamentali e strumenti di controllo dell'immigrazione, in I percorsi giuridici dell'integrazione. Migranti e titolari di protezione internazionale tra diritto dell'Unione e ordinamento italiano, G. Caggiano (a cura di), Torino 2014, pp.5-18; F. De Vittor, Il diritto di traversare il Mediterraneo...o quantomeno di provarci e, sotto altri profili, L. Masera, Il "caso Lampedusa": una violazione sistemica del diritto alla libertà personale, entrambi in Diritti umani e diritto internazionale, 2014, p. 63 ss. e 83 ss.

9. Cfr. Decision of the European Ombudsmam closing own-initiative inquiry OI/5/2012/BEH-MHZ concerning the European Agency for the Management of Operational Cooperation at the External Borders of the Member States of the European Union (Frontex).

10. Regolamento (UE) n. 656/2014 del Parlamento europeo e del Consiglio del 15.5.2014 recante norme per la sorveglianza delle frontiere marittime esterne nel contesto della cooperazione operativa coordinata dall'Agenzia europea per la gestione della cooperazione operativa alle frontiere esterne degli Stati membri dell'Unione europea in GUUE L 189 del 27.6.2014, p. 93 ss.

11. Ibidem, art. 4. Una simile disposizione era presente anche nel regolamento $n$. 1168/2011 che modificava il regolamento istitutivo di Frontex, il n. 2007/2004. 
Premesso un esame sul quadro normativo applicabile alle attività di Frontex, l'articolo affronterà sia i temi relativi al diritto applicabile, sia quelli relativi alla responsabilità, analizzando criticamente le norme del regolamento 656/2014 soprattutto in relazione all'obbligo di non respingimento e alle operazioni di ricerca e soccorso in mare.

\section{Segue. Alcune considerazioni generali sulle norme in materia di di- ritti umani applicabili nel controllo alle frontiere esterne dell'UE}

Prima di procedere all'analisi vera e propria del diritto applicabile, è necessario premettere alcune considerazioni di ordine generale sul quadro normativo nell'ambito del quale si collocano le operazioni di controllo alle frontiere esterne coordinate da Frontex.

L'analisi che segue parte dal presupposto che le norme sui diritti umani applicabili siano quelle che si impongono agli Stati membri dell'UE in virtù degli obblighi internazionali da essi contratti. Una tale affermazione vale anche ora che la Carta dei diritti fondamentali dell'UE è assunta al rango di norma primaria e ha natura vincolante. ${ }^{12}$ La tutela offerta dalla Carta, infatti, affonda le sue radici negli obblighi internazionali degli Stati membri ${ }^{13} \mathrm{e}$, in particolare, deve essere armonizzata con quella offerta nell'ambito della CEDU; ${ }^{14}$ l'interpretazione che la Corte EDU dà delle disposizione della CEDU è quindi parte dei principi generali del diritto dell'UE. ${ }^{15}$

Accanto ad essi, occorre anche considerare tutto l'insieme di norme secondarie dell'UE preposte alla regolazione dei flussi migratori. ${ }^{16}$ Tra que-

12. Carta dei diritti fondamentali dell'Unione europea, in GUUE C 83 del 30.3.2010, p. 389 ss.

13. Ibidem, Preambolo: «La presente Carta riafferma, nel rispetto delle competenze e dei compiti dell'Unione e del principio di sussidiarietà, i diritti derivanti in particolare dalle tradizioni costituzionali e dagli obblighi internazionali comuni agli Stati membri [...]».

14. Ibidem, art. 52, co. 3: «Laddove la presente Carta contenga diritti corrispondenti a quelli garantiti dalla Convenzione europea per la salvaguardia dei diritti dell'uomo e delle libertà fondamentali, il significato e la portata degli stessi sono uguali a quelli conferiti dalla suddetta Convenzione. La presente disposizione non preclude che il diritto dell'Unione conceda una protezione più estesa».

15. Ciò che è ribadito anche nell' art. 6 TUE, co. 3: «I diritti fondamentali, garantiti dalla Convenzione europea per la salvaguardia dei diritti dell'uomo e delle libertà fondamentali e risultanti dalle tradizioni costituzionali comuni agli Stati membri, fanno parte del diritto dell'Unione in quanto principi generali».

16. Sul punto si rimanda a F. Cherubini, L'asilo dalla Convenzione di Ginevra al diritto dell'Unione europea, Bari 2012, p. 158 ss. 


\section{A. Spagnolo}

ste possiamo ricordare le norme di cui al c.d. "sistema Dublino", ${ }^{17}$ la direttiva "procedure" 18 e la direttiva "qualifiche": ${ }^{19}$ l'impatto di tali norme sulla determinazione e il rispetto dei diritti dei migranti è visibile ictu oculi.

L'attività di Frontex è ora inserita nel contesto più ampio della cooperazione nel controllo alle frontiere esterne, il cui coordinamento è ora disciplinato nel regolamento istitutivo di EUROSUR, ${ }^{20}$ che, come si vedrà, è incentrato sul rispetto dei diritti umani.

La prospettiva in cui si colloca il presente scritto è quella di una lettura integrata delle norme appena citate e di quelle poste a tutela dei diritti umani di origine convenzionale e di fonte di produzione esterna all'UE. La materia, infatti, è terreno di una necessaria cross-fertilization tra norme giuridiche che si influenzano reciprocamente.

Ad esempio, l'obbligo di non respingimento, ${ }^{21}$ che è la pietra angolare di tutto il sistema di protezione, incluso, come vedremo, nell'art. 4 del nuovo regolamento 656/2014, è affermato nella Carta dei diritti fondamentali dell'UE, quando vi sia, per un individuo, il «rischio serio di essere sottoposto alla pena di morte, alla tortura o ad altre pene o trattamenti inumani o degradanti». ${ }^{22}$

Esso è poi tutelato da una pluralità di norme di natura convenzionale, tra cui, in via diretta, l'art. 33 della Convenzione delle Nazioni Unite sullo sta-

17. Attualmente disciplinato dal c.d. regolamento Dublino III. V. regolamento (UE) $n$. 604/2013 del Parlamento europeo e del Consiglio del 26.6.2013 che stabilisce i criteri e i meccanismi di determinazione dello Stato membro competente per l'esame di una domanda di protezione internazionale presentata in uno degli Stati membri da un cittadino di un Paese terzo o da un apolide (rifusione), in GUUE L 180/31 del 29.6.2013, p. 1 ss.

18. Direttiva (UE) n. 32/2013 del Parlamento europeo e del Consiglio del 26.6.2013, recante procedure comuni ai fini del riconoscimento e della revoca dello status di protezione internazionale (rifusione), in GUUE L 180 del 29.6.2013, p. 1 ss.

19. Direttiva (UE) n. 95/2011 del Parlamento europeo e del Consiglio del 13.12.2011 recante norme sull'attribuzione, a cittadini di Paesi terzi o apolidi, della qualifica di beneficiario di protezione internazionale, su uno status uniforme per i rifugiati o per le persone aventi titolo a beneficiare della protezione sussidiaria, nonché sul contenuto della protezione riconosciuta, in GUUE L 337 del 20.12.2011, p. 9 ss.

20. Regolamento (UE) n. 1052/2013 del Parlamento europeo e del Consiglio, del 22.10.2013, che istituisce il Sistema europeo di sorveglianza delle frontiere (EUROSUR), GUUE L 295 del 6.11.2013, p. 11 ss.

21. In dottrina, sull'obbligo di non respingimento cfr. S. Trevisanut, The principle of non refoulement at sea and the effectiveness of asylum protection, in Max Planck Yearbook of United Nations Law, 2008, p. 205 ss.; F. Salerno, L'obbligo internazionale di non refoulement dei richiedenti asilo, in Diritti umani e diritto internazionale, 2010, p. 287 ss.

22. Carta dei diritti fondamentali dell'Unione europea, cit., art. 19, co. 2. 
tus dei rifugiati del 1951 (Convenzione di Ginevra) ${ }^{23}$ e la Convenzione contro la tortura, ${ }^{24}$ ancorché con contenuto diverso, e, in via indiretta, la Convenzione europea dei diritti umani (CEDU) ${ }^{25}$ e il Patto sui diritti civili e politici. ${ }^{26}$

L'obbligo di non respingimento, oltre a essere specificamente pensato per la protezione dei rifugiati, è considerato un corollario del divieto di tortura e trattamenti inumani e degradanti sancito nelle norme convenzionali in materia di diritti umani. ${ }^{27}$ Essendo il divieto di tortura una norma di jus cogens,${ }^{28}$ l'obbligo di non respingimento, in quanto parte integrante della norma, assume un carattere "assoluto". ${ }^{29}$

Chiaramente, l'ambito di applicazione della Convenzione di Ginevra non coincide con quello delle Convenzioni in materia dei diritti umani: la prima si applica ai cosiddetti "statutory refugees", definiti nell'art. 1 della Convenzione stessa; ${ }^{30}$ le norme in materia di diritti umani non si applicano in-

23. Convenzione relativa allo status dei rifugiati, 28.7.1951, 189 UNTS 137.

24. Convenzione contro la tortura e altre pene o trattamenti crudeli, inumani o degradanti, 10.12.1984, 1465 UNTS 85.

25. Convenzione europea per la salvaguardia dei diritti umani e delle libertà fondamentali, 4.11.1950, 213 UNTS 222.

26. Patto internazionale sui diritti civili e politici, 16.12.1966, 999 UNTS 171.

27. L'obbligo di non respingimento è incluso nell'art. 3 della Convenzione contro la tortura. Diversamente, né il Patto sui diritti civili e politici, né la CEDU menzionano espressamente il divieto di refoulement. Questo è però ricavabile dalla ricca giurisprudenza, specialmente della Corte EDU, sull'obbligo di non respingimento di individui che rischino di subire, nel Paese di destinazione, trattamenti inumani e degradanti e/o atti di tortura. Si veda, per tutti, S. Trevisanut, The principle of non refoulement at sea..., cit., pp. 212-215. Vedasi anche F. Lenzerini, Asilo e diritti umani, Milano 2009, p. 378 ss.

28. Così si era espressa la Corte EDU nel caso Al-Adsani c. Regno Unito (ricorso $\mathrm{n}$. 35763/97, sentenza del 21.11.2001, par. 60-61). Sul punto, si rimanda ex multis a G. Cataldi, Osservazioni sulla giurisprudenza della Corte europea dei diritti umani in materia di tortura, in Diritti umani e diritto internazionale, 2008, p. 51 ss. e ai riferimenti ivi citati, in partic. a p. 54.

29. È quindi escluso che gli Stati possano giustificare l'allontanamento di un individuo con esigenze di sicurezza nazionale, quando l'individuo stesso rischi di subire tortura o trattamenti inumani e degradanti nel Paese di destinazione. V. A. Gianelli, Il carattere assoluto dell'obbligo di non refoulement: la sentenza Saadi della Corte europea dei diritti umani, in Rivista di diritto internazionale, 2008, p. 449 ss.

30. Il termine "tatutory refuge" si applica a quelle persone che «owing to well founded fear of being persecuted for reasons of race, religion, nationality, membership of a particular social group or political opinion, is outside the country of his nationality and is unable or, owing to such fear, is unwilling to avail himself of the protection of that country; or who, 


\section{A. Spagnolo}

vece a una categoria così ristretta di persone, potendosi configurare l'obbligo di non respingimento allorquando un individuo rischi di subire la violazione di determinati diritti.

È proprio la prassi degli organi preposti al controllo del rispetto dei diritti enunciati nelle Convenzioni appena citate ad aver contribuito ad affermare alcuni principi fondamentali nella determinazione e nella definizione degli obblighi a carico degli Stati nella gestione dei flussi migratori. Risulta, quindi, di tutta evidenza come le norme dell'UE debbano necessariamente essere interpretate alla luce di una giurisprudenza così evoluta; ciò deve poter valere anche per altre ipotesi di diritti.

Basti pensare, inter alia, al ruolo che ha avuto la giurisprudenza della Corte europea dei diritti umani nell'enunciare principi che, infine, hanno portato l'UE a modificare la sua legislazione. ${ }^{31}$

\section{L'ambito di applicazione delle norme convenzionali in materia di diritti umani. In particolare, l'applicazione extraterritoriale dell'obbligo di non respingimento}

Senza dubbio all'interno dello spazio territoriale dell'UE si devono considerare applicabili tutte le norme convenzionali che consacrano l'obbligo di non respingimento. In primis il riferimento deve necessariamente essere all'art. 33 della Convenzione di Ginevra. ${ }^{32}$ Le norme qui contenute sono pacificamente considerate applicabili nell'ambito dei confini nazionali degli Stati parte della Convenzione inclusi, ovviamente, i confini "marittimi". 33

Proprio con specifico riferimento all'estensione nelle acque territoriali degli Stati parte della Convenzione di Ginevra è da segnalarsi la prassi di alcuni di essi - nello specifico Australia e Francia - di escludere determina-

not having a nationality and being outside the country of his former habitual residence as a result of such events, is unable or, owing to such fear, is unwilling to return to it».

31. Si veda principalmente la sentenza della Corte EDU nel caso MSS. cfr. Corte europea dei diritti umani, MSS c. Belgio e Grecia, ricorso n. 30696/09, sentenza del 21.1.2011 che ha indotto l'UE a modificare il "sistema Dublino".

32. Sulla protezione dei rifugiati si vedano inter alia J. H. Hathaway: The Rights of Refugees under International Law, Cambridge, 2005; G.S. Goodwin-Gill and J. McAdam, The Refugee in International Law, Oxford 2007; Id., The International Law of Refugee Protection in The Oxford Handbook of Refugee and Forced Migration Studies, Oxford 2014, E. Fiddian-Qasmiyeh, G. Loescher, K. Long, N. Sigona (eds).

33. Sul punto, per un'analisi estesa in merito all'applicazione dell'obbligo di non respingimento in acque territoriali, cfr. S. Trevisanut, The principle of non refoulement at sea..., cit., pp. 219-230. 
te aree dalla protezione di cui alla Convenzione di Ginevra. Una siffatta prassi è stata aspramente criticata ${ }^{34} \mathrm{e}$, per quanto riguarda la Francia, è stata oggetto di un giudizio censore da parte della Corte europea dei diritti umani nel caso Armuur. ${ }^{35}$

Si può quindi pacificamente affermare che gli Stati europei - rectius, tutti gli Stati - non possano creare delle "zone franche" nelle quali ritenere non applicabili determinate norme internazionali poste a tutela dei diritti umani, incluse quelle relative alla tutela dei rifugiati, in particolare l'obbligo di non respingimento.

L'applicazione di tale obbligo è meno pacifica in acque internazionali. ${ }^{36}$

Ripartendo, come è necessario fare, dalla Convenzione di Ginevra, occorre fin da subito evidenziare come vi sia stata una certa prassi negativa rispetto all'applicazione extraterritoriale - e, quindi, in alto mare - dell'art. 33. Si fa qui riferimento, principalmente, alla nota sentenza della Corte suprema statunitense nel caso Sale c. Haitian Ctrs. Council, ${ }^{37}$ con la quale è stato ribadito il limite dell' applicazione territoriale della Convenzione di Ginevra.

A detta prassi si è immediatamente opposta, peraltro sulle medesime circostanze fattuali, una pronuncia della Commissione interamericana dei diritti umani. ${ }^{38}$ Un simile orientamento è poi andato a consolidarsi in seno all'Alto Commissariato delle Nazioni Unite (ACNUR), ${ }^{39}$ le cui prese di posizione possono essere considerate utili strumenti interpretativi.

34. M. O'Sullivan, Withdrawing Protection under Article 1 C(5) of the 1951 Convention: Lessons from Australia, in International Journal Refugee Law, 2008, p. 586 ss., in partic. p. 610.

35. Corte europea dei diritti umani, Amuur c. Francia, ricorso n. 19776/92, sentenza del 25.6.1996, par. 52-53.

36. A. Terrasi, op. cit., pp. 600-601; T. Gammeltoft-Hansen, The Externalisation of European Migration Control and the Reach of International Refugee Law, in European Journal of Migration Law, 2010, p. 1 ss.

37. Sale v. Haitian Ctrs. Council, 509 US 155, 156 (USSC 1993). Cfr. S. Goodwin-Gill, The Haitian Refoulement Case: A Comment, in International Journal of Refugee Law, 1994, p. 103 ss.

38. Commissione inter-americana dei diritti umani, The Haitian Centre for Human Rights e altri c. Stati Uniti, caso n. 10.676, decisione del 13.3.1997.

39. Alto Commissariato delle Nazioni Unite per i Rifugiati (ACNUR), Parere consultivo sull'applicazione extraterritoriale degli obblighi di non-refoulement derivanti dalla Convenzione relativa allo status dei rifugiati del 1951 e dal suo Protocollo del 1967, Ginevra, 26.1.2007, disponibile in italiano sul sito internet dell'ACNUR alla pagina www.unhcr.it.

40. Di cui tuttavia non va sottovalutato il valore, se non altro ai fini interpretativi. Si veda, proprio con riferimento al ruolo dell'ACNUR, I. Venzke, How interpretation makes international law. On semantic chance and normative twists, Oxford 2012, pp. 72-134. 


\section{A. Spagnolo}

L'applicazione extraterritoriale dell'obbligo di non respingimento sembra poi essere supportata da un'ermenèutica del testo della Convenzione di Ginevra: a un siffatto risultato si perviene ugualmente anche adottando metodi interpretativi diversi. ${ }^{41}$

Secondo alcuni Autori, infatti, sarebbe la stessa interpretazione letterale della norma in questione a suggerire l'estensione dell'ambito di applicazione. Ad essere menzionate nell'art. 33, infatti, sarebbero due condotte, "expel" e "return" o "refouler" nella versione francese, la seconda delle quali - l'obbligo di non refoulement, appunto - necessariamente implicherebbe un'applicazione extraterritoriale. ${ }^{42}$ Altri Autori affermano che a sostenere l'applicazione extraterritoriale dell'obbligo sia un'interpretazione letterale del termine "whatsoever" contenuto nella versione inglese della norma. ${ }^{43}$

Anche a voler dare alla lettera della norma un significato diverso da quello proposto dalla dottrina - ciò che ha fatto la Corte suprema statunitense nel caso Sale - l'applicazione extraterritoriale sembra essere confortata da un'interpretazione della Convenzione di Ginevra finalizzata a tutelare le vittime delle persecuzioni che darebbero titolo a richiedere lo status di rifugiato. ${ }^{44}$

In questa direzione deporrebbe anche un'interpretazione dinamica della Convenzione, in armonia con l'evoluzione della giurisprudenza e della prassi in materia di tutela dei diritti umani. ${ }^{45}$

Non si può fare a meno di notare che l'evoluzione della giurisprudenza legata all'applicazione extraterritoriale delle norme in materia di diritti umani, in particolare della CEDU, abbia contribuito a consolidare l'applicazione dell'art. 33 della Convenzione di Ginevra al di fuori dei confini nazionali. ${ }^{46}$

41. Convenzione di Vienna sul diritto dei Trattati, 23.5.1969, 1155 UNTS 331, art. 31. Tale norma costituisce la c.d. "regola generale d'interpretazione", che impone innanzitutto di seguire un metodo obbiettivistico, che tenga, quindi, conto dell'interpretazione letterale del testo (cfr. B. Conforti, Diritto internazionale, X ed., Napoli 2014, pp. 112-114). Accanto ad esso, e soprattutto nell'ambito dell'interpretazione dei Trattati sui diritti umani, si fa solitamente ricorso a un approccio teleologico, che tenga conto del particolare oggetto di questi Trattati, e a un approccio dinamico e contestuale, che tenga conto dell'evoluzione delle norme in materia di diritti umani (in generale e per tutti si rimanda a U. Villani, Dalla Dichiarazione universale alla Convenzione europea dei diritti dell'uomo, Bari 2012).

42. T. Gammeltoft-Hansen, op. cit., p. 10.

43. A. Fischer-Lescano, T. Löhr, T. Tohidipur, op. cit., pp. 268-269; S. Goodwin-Gill, The Haitian Refoulement Case: A Comment, cit., p. 103.

44. A. Fischer-Lescano, T. Löhr, T. Tohidipur, op. cit., p. 270.

45. A. Terrasi, op. cit., pp. 601-602; T. Gammeltoft-Hansen, op.cit., pp. 11-12.

46. T. Gammeltoft-Hansen, op.cit., p. 22. Alle medesime conclusioni addiveniva l'Alto Commissariato delle Nazioni Unite per i Rifugiati (ACNUR), Parere consultivo 
$\mathrm{Ne}$ deriva che l'obbligo di non respingimento viene condizionato all'esercizio, da parte degli Stati cui si impone l'obbligo stesso, della propria giurisdizione.

Sul punto, la giurisprudenza della Corte di Strasburgo ha da tempo consolidato alcuni principi che governano l'applicabilità dei diritti tutelati dalla CEDU al di fuori del c.d. espace juridique degli Stati parte della Convenzione. ${ }^{48}$ La Corte ha, invero, spesso oscillato adottando, a partire dal celebre caso Loizidou, ${ }^{49}$ orientamenti spesso in netto contrasto. Con la pronuncia nel caso Al-Skeini, nel 2011, la Corte ha finalmente provato a "sistemare" la materia, sancendo come l'applicazione extraterritoriale della CEDU sia possibile a condizione che lo Stato eserciti una forma di controllo sulle vittime delle condotte poste in essere al di fuori del proprio territorio, ${ }^{50}$ anche in alto mare. ${ }^{51}$

sull'applicazione extraterritoriale degli obblighi di non-refoulement derivanti dalla Convenzione relativa allo status dei rifugiati del 1951 e dal suo Protocollo del 1967, cit., par. 43, p. 19.

47. Intesa nel senso di "jurisdiction”, così come riportata nei Trattati internazionali in materia di diritti umani e, per quanto riguarda la CEDU, nell'art. 1. Cfr. R. Sapienza, Art. 1, in Commentario breve alla Convenzione europea dei diritti dell'uomo, S. Bartole, P. De Sena, V. Zagrebelsky (a cura di), Padova, 2012, p. 13 ss.

48. In generale sul tema si vedano P. De Sena, La nozione di giurisdizione statale nei Trattati sui diritti umani, Torino, 2002. Per un'analisi della giurisprudenza più recente cfr. D. Russo, Nuove pronunce sull'applicazione extraterritoriale degli obblighi previsti dai Trattati sui diritti umani, in Rivista di diritto internazionale 2009, p. 271 ss.; M. Milanovic, Extraterritorial Application of Human Rights Treaties, Oxford, 2011. Si vedano anche i contributi pubblicati in Extraterritorial Application of Human Rights Treaties, in F. Coomans, M.T. Kamminga (eds.), Anversa, 2004. Con specifico riferimento alla recente giurisprudenza della Corte europea dei diritti umani cfr. R. Nigro, Notion of Jurisdiction in Article 1: future scenarios for the extraterritorial application of the European Convention on Human Rights, in Italian Yearbook of International Law 2011, p. 11 ss.; S. Besson, The Extraterritoriality of the European Convention on Human Rights: Why Human Rights Depend on Jurisdiction and What Jurisdiction Amounts to, in Leiden Journal of International Law, 2012, p. 857 ss.

49. Corte europea dei diritti umani, Loizidou c. Turchia, ricorso n. 15318/89, sentenza (preliminary objections) del 23.3.1995, par. 62 e sentenza del 18.12.1996, par. 56.

50. Corte europea dei diritti umani, Al-Skeini c. Regno Unito, ricorso n. 55721/07, sentenza del 7.7.2011, par. 149. Cfr. R. Nigro, I casi Al-Skeini e Al-Jedda dinanzi alla Corte europea dei diritti umani, in Diritti umani e diritto internazionale, 2012, p. 616 ss., in partic. p. 618.

51. Cfr. Corte europea dei diritti umani, Medvedyev c. Francia, ricorso n. 3394/03, sentenza del 29.3.2010, su cui cfr. S. Trevisanut, Lotta al traffico di sostanze stupefacenti e tutela dei diritti umani in mare: il caso Medvedyev dinanzi alla Corte europea dei diritti umani, in Diritti umani e diritto internazionale, 2009, p. 192 ss. 


\section{A. Spagnolo}

Non può, poi, essere taciuto l'ultimo orientamento della Corte europea dei diritti umani, che con la sentenza Hirsi ha affermato l'applicazione della Convenzione europea dei diritti umani nel contesto dei respingimenti operati dall'Italia verso la Libia in acque internazionali. ${ }^{52}$

In quest'ultimo caso, i Giudici di Strasburgo sono addivenuti a tale conclusione attraverso l'applicazione extraterritoriale dell'obbligo di non respingimento giustificato dalla presenza dei ricorrenti su navi battenti bandiera italiana e su cui si estende, pacificamente, la giurisdizione dello Stato italiano. ${ }^{53}$

Una così chiara e lineare linea interpretativa rende potenzialmente incerta l'applicabilità della CEDU in altri scenari, in cui, ad esempio, eventuali barconi vengano "scortati" senza che vi sia alcun contatto con i membri dell'equipaggio e senza che, va da sé, gli stessi vengano ospitati a bordo della nave dello Stato costiero. Ancora, il richiamo alla giurisdizione statale può essere di difficile argomentazione proprio nel caso delle operazioni coordinate da Frontex, in cui il personale può anche essere di diverse nazionalità, come nel caso delle squadre europee di guardia alle frontiere. ${ }^{54}$

Circostanze fattuali diverse da quelle del caso Hirsi, tuttavia, non implicano che lo Stato membro non possa comunque esercitare una forma di controllo sui migranti respinti.

A una siffatta conclusione si può addivenire ricorrendo a un approccio fondato sull'effettività che si poggia su una differenziazione tra obblighi negativi e obblighi positivi.

Per i primi, l'estensione della giurisdizione seguirebbe l'attribuzione della condotta allo Stato, rectius sarebbe sufficiente stabilire che lo Stato stes-

52. Corte europea dei diritti umani, Hirsi Jamaa e altri c. Italia, ricorso n. 27765/09, sentenza del 23.2.2012. Per un commento alla sentenza si vedano A. Liguori, La Corte europea dei diritti dell'uomo condanna l'Italia per i respingimenti verso la Libia del 2009: il caso Hirsi, in Rivista di diritto internazionale, 2012, p. 415 ss., in partic. p. 423; G. Cellamare, Brevi note sulla sentenza della Corte europea dei diritti dell'uomo nell'affare Hirsi Jamaa e altri c. Italia, in Studi sull'integrazione europea, 2012, p. 491 ss.; U. Villani, Osservazioni sulla sentenza della Corte europea nell'affare Hirsi Jamaa e sui problemi relativi alla sua esecuzione, in I diritti dell'uomo. Cronache e battaglie, 2012, n. 1, p. 9 ss.; cfr. anche A. Di Pascale, La sentenza Hirsi e altri c. Italia: una condanna senza appello della politica dei respingimenti, in questa Rivista, n. 1.2012, p. 85 ss.; N. Napoletano, La condanna dei "respingimenti" operati dall'Italia verso la Libia da parte della Corte europea dei diritti umani: molte luci e qualche ombra, in Diritti umani e diritto internazionale, 2012, p. 34.

53. I ricorrenti, infatti, erano stati fatti salire a bordo di navi battenti bandiera italiana, dove si sarebbero poi verificate le violazioni dei diritti umani per cui l'Italia è stata condannata.

54. In questo senso cfr. N. Napoletano, op. cit., p. 34. 
se, al momento della condotta, esercitando un controllo effettivo sulla vittima della violazione. ${ }^{55}$

Per i secondi, invece, sarebbe necessario un controllo più diffuso, che implichi anche una dimensione territoriale, non essendo sufficiente il mero controllo sul soggetto vittima della condotta. In altri termini, lo Stato deve ragionevolmente poter influire, con la sua condotta, sulla protezione delle potenziali vittime. ${ }^{56}$

L'obbligo di non respingimento, essendo di natura negativa, può quindi applicarsi al di fuori del territorio dello Stato in questione anche in assenza di un controllo diffuso sulla porzione di territorio - alto mare incluso - in cui si trova il migrante, potendo bastare che lo Stato eserciti un controllo sull'individuo.

E, invero, sembra che un tale controllo possa sussistere anche in assenza di contatto fisico con i migranti; a deporre in questo senso è la giurisprudenza della Corte EDU, in particolare nel caso Women on Waves ${ }^{57}$ Effettivamente, risulterebbe problematico, per uno Stato, negare la propria giurisdizione su individui nei confronti dei quali sta esercitando una forma di "potere pubblico", anche in assenza di un contatto fisico con essi. ${ }^{58}$

55. Cfr. M. Milanovic, Extraterritorial Application, cit., pp. 207-209; R. Sapienza, Art. 1, cit., p. 24. Prima ancora della pronuncia nel caso Al-Skeini che sembra rafforzare questa ipotesi, si esprimeva in questo senso P. De Sena, La nozione, cit., pp. 145-159, in partic. pp. 155-157. L'A. critica proprio l'approccio secondo cui vi sarebbe, nella giurisprudenza della Corte europea, un'assimilazione tra l'espansione della giurisdizione statale e l'imputazione della condotta allo Stato, evidenziando l'autonomia dei due fenomeni, nonché la tendenza del primo a condizionare il secondo. Ma si veda contra S. Besson, op. cit., pp. 878-879. Per questa Autrice al fine dell'applicazione extraterritoriale dei Trattati in materia di diritti umani sarebbe sempre necessario l'esercizio di una qualche forma di "potere normativo" da parte dello Stato la cui giurisdizione si suppone estendersi al di là del proprio territorio.

56. Cfr. J. Cerone, Minding the gap: Outlining KFOR Accountability in Post-Conflict Kosovo, in European Journal of International Law 2001, pp. 479-480; M. Milanovic, Extraterritorial Application, cit., p. 213. Sul punto si rinvia all'analisi di P. De Sena, La nozione, cit., pp. 220-223, nella parte in cui l'A. ricostruisce gli obblighi che gravano sugli Stati «in funzione dell'idoneità dei poteri da essi esercitati ad incidere [...] sul godimento dei $[\ldots]$ diritti» (p. 221).

57. Corte europea dei diritti umani, Women on waves e altri c. Portogallo, ricorso n. 31276/05, sentenza del 3.2.2009, par. 43. In commento alla sentenza si veda I. Papanicolopulu, Donne sulle onde: libertà di navigazione, libertà di espressione o libertà di circolazione?, in Diritti umani e diritto internazionale, 2010, p. 205 ss., in partic. pp. 207-208.

58. Si fa qui riferimento alla nozione di "public powers" adottata dalla Corte EDU nella pronuncia nel caso Al-Skeini (cit., par. 149). 


\section{A. Spagnolo}

Più complesso sembra essere il discorso relativamente all'obbligo di ricerca e salvataggio in mare aperto che invece è di natura positiva. Sul punto, su cui si tornerà più avanti, sembra che un minimo di controllo territoriale, nei termini che esporremo, sia necessario. ${ }^{59}$

\section{Il contenuto dell'obbligo di non respingimento così come recepito nel regolamento $\mathrm{n}$. 656/2014}

Come anticipato nelle premesse, il tema del rispetto dei diritti umani si trova riflesso nelle importanti innovazioni normative introdotte con il regolamento n. 656/2014.

Occorre senz'altro notare come l'attuale formulazione dell'art. 4 del regolamento 656/2014 sia la risposta alle numerose critiche ricevute dall'Agenzia con riguardo alle incertezze in merito all'applicazione del principio di non respingimento.

Senza dubbio, l'introduzione di un articolo ad hoc nel nuovo regolamento Frontex che imponga agli Stati partecipanti il rispetto dell'obbligo di non respingimento sembra essere prima facie una risposta adeguata alle pressioni ricevute dall'Agenzia in questa direzione.

La formulazione del co. 1 dell'art. 4 del regolamento 656/2014 è molto ampia ed è tale di ritenere applicabile l'obbligo di non respingimento in tutti i casi in cui vi sia un rischio grave di violazione dei diritti umani nel Paese di destinazione. ${ }^{60}$

La "storia" della redazione di questa disposizione dimostra come gli organi legislativi dell'UE abbiano recepito alcune indicazioni portate all'attenzione della Commissione europea dall'ACNUR. ${ }^{61}$ La prima formu-

59. Si rimanda a infra, par. 5 .

60. Regolamento (UE) n. 656/2014, cit., art. 4: «Nessuno può, in violazione del principio di non respingimento, essere sbarcato, costretto a entrare, condotto o altrimenti consegnato alle autorità di un Paese in cui esista, tra l'altro, un rischio grave di essere sottoposto alla pena di morte, alla tortura, alla persecuzione o ad altre pene o trattamenti inumani o degradanti, o in cui la vita o la libertà dell'interessato sarebbero minacciate a causa della razza, della religione, della cittadinanza, dell'orientamento sessuale, dell'appartenenza a un particolare gruppo sociale o delle opinioni politiche dell'interessato stesso, o nel quale sussista un reale rischio di espulsione, rimpatrio o estradizione verso un altro Paese in violazione del principio di non respingimento».

61. Alto Commissariato delle Nazioni Unite per i Rifugiati (ACNUR), UNHCR comments on the Commission proposal for a Regulation of the European Parliament and of the Council establishing rules for the surveillance of the external sea borders in the context of operational cooperation coordinated by the European Agency for the Management of Opera- 
lazione dell'art. 4, infatti, menzionava solo il rischio grave di subire, nel Paese di destinazione, atti di tortura o trattamenti inumani e degradanti; ${ }^{62}$ quella attuale prevede un'elencazione più ampia che dovrebbe poter estendere la protezione anche a coloro i quali corrano un rischio grave di subire persecuzioni e/o gravi violazioni dei diritti umani su base discriminatoria. ${ }^{63}$

L'applicazione dell'obbligo di non respingimento, così elaborato, sembra non avere limiti in relazione alle varie operazioni di cui al regolamento, almeno stando al dettato normativo del co. 7 , sempre dell'art. $4 .{ }^{64} \grave{E}$ indiscutibile, quindi, l'applicazione nell' ambito di intercettazioni in acque internazionali, dove è ancora prevista una forma residuale di respingimento: le unità partecipanti sono autorizzate $\mathrm{a}$ «condurre il natante o le persone $\mathrm{a}$ bordo in un Paese terzo o altrimenti consegnare il natante o le persone a bordo alle autorità di un Paese terzo».

tional Cooperation at the External Borders of the Member States of the European Union (Frontex) COM 2013(197) final, aprile 2014.

62. Commissione europea, Proposal for a REGULATION OF THE EUROPEAN PARLIAMENT AND OF THE COUNCIL establishing rules for the surveillance of the external sea borders in the context of operational cooperation coordinated by the European Agency for the Management of Operational Cooperation at the External Borders of the Members States of the European Union, $\operatorname{COM(2013)~} 197$ final, 12.4.2013, p. 13: «No person shall be disembarked in, or otherwise handed over to the authorities of a country where there is a serious risk that such person would be subjected to the death penalty, torture or other inhuman or degrading treatment or punishment or from which there is a serious risk of expulsion, removal or extradition to another country in contravention of the principle of nonrefoulement».

63. Su impulso proprio dell'ACNUR la norma è stata modificata ampliando la tutela originariamente prevista (si veda nota precedente) a tutti i casi di discriminazione, anche su base sessuale. Proprio sotto quest'ultimo profilo è interessante notare come una recente sentenza della Corte di giustizia dell'UE ponga diversi limiti - una sorta di black list - agli Stati nel trattare con migranti omosessuali: vi sarebbero determinate domande che non possono essere loro rivolte al fine di non ledere il diritto al rispetto alla vita privata e famigliare del soggetto in questione. La pronuncia è destinata a far parlare di sé e le intersezioni con la tutela dei diritti umani nell'ambito dell'attività di Frontex sembrano inevitabili. Cfr. Corte di giustizia dell'Unione europea, $A, B, C$, cause riunite n. C-148/13, C-149/13, C-150/13, sentenza del 2.12.2014.

64. «Il presente articolo si applica a tutte le misure adottate dagli Stati membri o dall'Agenzia a norma del presente regolamento».

65. Regolamento (UE) 656/2014, cit., art. 7, co. 2, lett. c), che disciplina il caso in cui siano riscontrate prove a conferma del sospetto che un natante sia impiegato per il traffico di migranti. Sulla legittimità di questa disposizione, che pare dubbia, si tornerà in sede di conclusioni in questo stesso paragrafo. 


\section{A. Spagnolo}

La disposizione appena citata desta preoccupazioni, in quanto l'operazione ivi descritta rischia di non essere soggetta alle norme procedurali del Sistema europeo di asilo. La direttiva "procedure", infatti, è limitata, nel suo campo di applicazione, alle sole «domande di protezione internazionale presentate nel territorio, compreso alla frontiera, nelle acque territoriali o nelle zone di transito degli Stati membri». ${ }^{66}$ Questa disposizione appare quanto meno di dubbia legittimità e rischia di "sollevare" gli agenti di un'operazione Frontex da determinati obblighi procedurali nei confronti di migranti intercettati in alto mare, ciò che è ancora più grave nei casi in cui le autorità coinvolte decidano di respingere un natante.

Più in generale, sembra che l'art. 7, co. 2, lett. c) possa consentire un'analisi non accurata delle istanze personali degli individui coinvolti, ciò che, oltre a essere discriminatorio per $s e,{ }^{67}$ non è allineato con gli standard dettati dalla Corte EDU in materia di valutazione del rischio, come si dimostrerà alla fine del presente paragrafo.

Viene poi ancora da chiedersi se le operazioni previste nel regolamento $656 / 2014$ riflettano al meglio tutto il ventaglio dei possibili scenari operativi in cui possa trovarsi coinvolto uno Stato membro dell'UE partecipante a un'operazione Frontex.

Non vi è menzione, infatti, delle operazioni che Frontex conduce nelle acque territoriali di Paesi terzi, pur essendo questa una prassi consolidata e mai abbandonata dell'Agenzia ${ }^{68}$ sebbene sia stata duramente criticata dal Parlamento europeo. ${ }^{69}$

66. Direttiva (UE) n . 32/2013, cit., art. 3.

67. F. De Vittor, op. cit., p. 75. Quanto detto si fonda sull'interpretazione che la Corte EDU ha fornito dell' art. 4 del Protocollo 4 della CEDU, che impone il divieto di espulsioni collettive. Nella sentenza Hirsi, infatti, la Corte di Strasburgo enuncia un importante principio in virtù del quale sarebbe discriminatorio consentire ai migranti "via terra" di poter rivolgere le proprie domande di protezione ad autorità competenti, mentre ai migranti "via mare", ciò sarebbe impedito, essendo considerati autorità competenti i militari presenti sulle navi che intercettano i migranti stessi. Ancora F. De Vittor, op. cit., p. 74.

68. Per una panoramica del fenomeno si vedano M. Fink, Frontex working arrangements: legitimacy and human rights concerns regarding "technical relationship", in Merkourios. Utrecht Journal of International and European Law, 2012, p. 20 ss.; F. Cherubini, La cooperazione fra Unione europea e Paesi del Nordafrica nella lotta all'immigrazione irregolare, in Atlante geopolitico del Mediterraneo, F. Anghelone, A. Ungari (a cura di), Roma, 2014, p. 15 ss.

69. E. Guild, S. Carrera, L. den Hertog, J. Parkin, Implementation of the EU Charter of Fundamental Rights and its impact on EU home affairs agencies. Frontex, Europol and European Asylum Support Office, Bruxelles 2011, p. 11: «The European Parliament should call upon Frontex to no longer conduct any joint operation in the maritime territory of third 
Ad aprile 2013 - ultimo dato disponibile sul sito internet dell'Agenzia - i c.d. "Working arrangements" conclusi con Stati terzi sono 27; per non menzionare quelli conclusi da Stati membri dell'UE uti singuli, come nel caso della Spagna in occasione dell'operazione Hera II. ${ }^{70}$

Nel contenuto di tali accordi - invero alquanto generici - non è mai esplicitato il coinvolgimento di Frontex in operazioni nelle acque territoriali dello Stato terzo contraente, né il consenso di quest'ultimo a quel fine; è solamente prevista una forma di "stretta collaborazione" con lo Stato in questione. ${ }^{71}$ Ciononostante, è pacifico che siffatti accordi ammettano che le operazioni di Frontex si svolgano nelle acque territoriali di Stati terzi ${ }^{72}$ il cui consenso deve ritenersi implicito.

In siffatte circostanze, vi è da domandarsi se l'obbligo di non respingimento possa estendersi anche nelle acque territoriali degli Stati terzi in cui opera Frontex, così colmando una lacuna normativa di siffatti accordi, che non sembrano considerare la tutela dei diritti umani. ${ }^{73}$

La risposta sembra doversi dare in senso positivo. In primis, la possibilità di condurre operazioni di controllo nelle acque territoriali di Stati terzi è prevista nel "Codice frontiere Schengen" del 2006. ${ }^{74}$ In quanto regolate da una norma di diritto dell'UE, le operazioni condotte nel territorio di Stati terzi devono rispettare i principi dell'Unione stessa, tra cui vi è, come visto nel paragrafo 2 di questo scritto, il rispetto dei diritti umani.

Anche ai sensi del diritto internazionale vi devono essere pochi dubbi: la giurisdizione dello Stato, e con essa gli obblighi convenzionali in materia di diritti umani, si estendono pacificamente sul territorio di Stati terzi, quando questi ultimi abbiano prestato il consenso. ${ }^{75}$

states, as the consistency of this practice is not only questionable with respect to the rule of law principles of legal certainty and accountability, but it is also at odds with fundamental rights foreseen in the EU Charter».

70. Per un' analisi degli accordi bilaterali siglati dalla Spagna, e sui quali si è basata l'operazione Hera II, si rimanda a P. G. Andrade, Extraterritorial Strategies to Tackle Irregular Immigration by Sea: A Spanish Perspective, in B. Ryan, V. Mitsilegas (eds.), op. cit., p. 318 ss.

71. Si rimanda ancora, sul punto a F. Cherubini, op. cit., pp. 31-33.

72. Si veda in questo senso A Baldaccini, op. cit., p. 245. Nella medesima direzione anche un Report presentato dinanzi alla Camera dei Lord: House of Lords, Frontex: The EU External Borders Agency. Report with Evidence, HL Paper 60 2008, par. 148.

73. In questo senso M. Fink, op. cit., pp. 33-34.

74. Regolamento (CE) n. 562/2006 del Parlamento europeo e del Consiglio del 15.3.2006 che istituisce un codice comunitario relativo al regime di attraversamento delle frontiere da parte delle persone (codice frontiere Schengen), in GUCE L 150, p. 1 ss., Allegato V, p. 3.1.1.

75. R. Sapienza, L'applicazione extraterritoriale dello Human Rights Act: la decisione della Camera dei Lords nel caso Al-Skeini, in Diritti umani e diritto internazionale, 2008, p. 154. 


\section{A. Spagnolo}

È chiaro, tuttavia, che l'assenza, nel regolamento 656/2014, di un qualsiasi riferimento alla tutela dell'obbligo di non respingimento nelle acque territoriali di Stati terzi espone, fin dal principio, il nuovo assetto di Frontex a diverse critiche.

Dietro la prassi degli appena citati accordi si cela un altro pericolo. Nel co. 2 dell'art. 4 è menzionata la possibilità che la valutazione del rischio che un individuo subisca una violazione di cui al co. 1 del medesimo articolo sia fondata su «accordi e progetti in materia di immigrazione e asilo realizzati conformemente al diritto dell'Unione e con fondi dell'Unione».

Chiaramente l'accordo in sé non può essere sufficiente per provare che un Paese terzo sia sicuro. Sul punto, la giurisprudenza della Corte EDU è consolidata nel senso di ritenere che mere assicurazioni formali non bastino per esonerare gli Stati membri dalla CEDU da una verifica dell'effettività della tutela dei diritti umani negli Stati terzi verso cui un individuo viene respinto. ${ }^{76}$

Il tema del luogo di sbarco riveste, evidentemente, un ruolo cruciale nelle operazioni coordinate da Frontex. In questa prospettiva, il regolamento $656 / 2014^{77}$ non pone rimedio a una lacuna sistemica della normativa europea in materia di immigrazione.

Il nuovo regolamento, infatti, dà per scontato che i Paesi partecipanti alle operazioni di controllo delle frontiere esterne siano "Paesi sicuri" ai sensi della normativa internazionale sui diritti umani.

Invero, la giurisprudenza, sia della Corte di Strasburgo, sia di quella di Lussemburgo, ha interpretato in maniera assai restrittiva il concetto di "Paese sicuro", giungendo a condannare - talvolta in maniera anche eclatante diversi Stati europei. ${ }^{78}$

76. Così si era espresso la Corte europea dei diritti umani nel caso Saadi con riferimento alle c.d. "assicurazioni diplomatiche", che non basterebbero a garantire che non vi sia alcun rischio che un individuo subisca determinate gravi violazioni dei diritti umani nel Paese che fornisce dette assicurazioni. Il principio mutatis mutandis può essere riproposto con riguardo agli eventuali accordi previsti dal co. 2 dell'art. 4 del regolamento 656/2014. Cfr. Corte europea dei diritti umani, Saadi c. Italia, ricorso n. 37201/06, sentenza del 28.2.2008, par. 147-148; in dottrina vedasi A. Tancredi, Assicurazioni diplomatiche e divieto "assoluto" di refoulement, in Diritti umani e diritto internazionale, 2010, p. 41 ss.

77. Che disciplina lo sbarco nell'art. 10.

78. Si veda la già citata sentenza M.S.S. c. Belgio e Grecia, ma anche la più recente Corte europea dei diritti umani, Tarakhel c. Svizzera, ricorso n. 29217/12, sentenza del 4.11.2014 con la quale la Corte EDU ha condannato la Svizzera che aveva emesso un ordine di espulsione verso l'Italia di richiedenti asilo sulla base del "Sistema Dublino". Per la Corte, che ha ingiunto alla Svizzera la sospensione del procedimento di espulsione, l'Italia non poteva essere considerato un Paese sicuro ai sensi della CEDU, rischiando, il ricorrente, di subire 
In ossequio al predetto orientamento il c.d. "sistema Dublino", che stabilisce criteri e meccanismi per la gestione, nell'UE, delle richieste di protezione internazionale, è stato più volte sindacato da Corti internazionali e nazionali, proprio sotto il profilo della tutela dei diritti umani e, nello specifico, della nozione di "Paese sicuro". 79

In particolare, è da tempo caduta la presunzione secondo cui gli Stati che fanno parte del "sistema Dublino" siano da considerarsi automaticamente come sicuri ${ }^{80}$ a favore di un orientamento che impone misure di scrutinio più penetranti al fine di verificare l'esistenza di garanzie effettive per i richiedenti asilo. ${ }^{81}$

Talvolta la giurisprudenza ha oscillato - nella definizione di tali misure tra due diversi approcci.

In ossequio al primo di essi, il fattore decisivo per valutare se uno Stato sia sicuro è stato identificato nelle carenze sistemiche del Sistema di accoglienza e gestione dei migranti. Depongono in questo senso le sentenze Hussein della Corte $\mathrm{EDU}^{82}$ ed $N S$, della Corte di giustizia dell' UE. ${ }^{83}$

trattamenti inumani e degradanti nel nostro Paese (par. 122 della sentenza). La sentenza è criticabile poiché si discosta nettamente da alcuni precedenti relativi proprio al Sistema italiano di accoglienza, che era stato valutato positivamente dalla Corte. Come vedremo più avanti, la sentenza Tarakhel, che, occorre evidenziarlo, è stata adottata con una maggioranza netta (14 a 3), è giustificata da un approccio metodologico diverso al concetto di "Paese sicuro".

79. Sul punto cfr. M. Marchegiani, Regolamento Dublino II e clausola di sovranità: il caso greco dinanzi all'Alto Commissariato per i rifugiati, in Diritti umani e diritto internazionale, 2010, p. 447 ss.

80. Inizialmente è stata la sentenza della Corte EDU nel caso K.R.S. c. Regno Unito (ricorso n. 32733/08, sentenza del 2.12.2008) a scardinare questo automatismo rilevando come l'art. 3.2 del regolamento 343/2003 (c.d. Dublino II) - la c.d. "clausola di sovranità", fosse strumento idoneo a sospendere i trasferimenti di richiedenti asilo verso Paesi non sicuri. La Corte EDU, nel caso di specie, non condannò il Regno Unito ritenendo non confutata la presunzione di conformità della Grecia al diritto dell'UE (par. 17).

81. Così, ancora, Corte europea dei diritti umani, M.S.S. c. Belgio e Grecia, cit., par. 342: «When they apply the Dublin Regulation, therefore, the States must make sure that the intermediary country's asylum procedure affords sufficient guarantees to avoid an asylumseeker being removed, directly or indirectly, to his country of origin without any evaluation of the risks he faces from the standpoint of Article 3 of the Convention».

82. Corte europea dei diritti umani, Hussein Diirshi e altri c. Paesi Bassi e Italia, ricorso n. 2314/10, decisione del 10.9.2013, par. 78: «it has not been shown to disclose a systemic failure to provide support or facilities catering for asylum seekers».

83. Corte di giustizia dell'UE, NS e altri, cause n. C-411/10 e C-493/10, sentenza del 21.12.2011, par. 94: «Discende da quanto sopra che, in situazioni come quelle oggetto dei procedimenti principali, al fine di permettere all'Unione e ai suoi Stati membri di rispettare i 


\section{A. Spagnolo}

Un secondo approccio, cui si possono ricondurre le sentenze Tarakhel della Corte EDU ${ }^{84}$ ed $E M$ della Corte suprema inglese, ${ }^{85}$ sembra, invece, dare maggior peso alla valutazione individuale della sussistenza di un concreto rischio che i migranti corrono nello Stato in questione di vedersi violati i più basilari diritti umani, oppure di essere perseguitati o peggio ancora di subire atti di tortura o trattamenti inumani o degradanti.

Nella prospettiva della protezione dei diritti umani deve ritenersi preferibile l'orientamento che meglio assicuri una tutela effettiva dei diritti dei migranti, in primis il diritto alla vita e il divieto di tortura. L'indagine sulla sussistenza o meno di carenze sistemiche o di violazioni sistematiche dei diritti umani negli Stati "di sbarco" è senz'altro necessaria, ma un suo eventuale esito negativo non può comunque far venir meno una valutazione del rischio individuale che corrono i migranti in quegli Stati. ${ }^{86}$

loro obblighi di tutela dei diritti fondamentali dei richiedenti asilo, gli Stati membri, compresi gli organi giurisdizionali nazionali, sono tenuti a non trasferire un richiedente asilo verso lo "Stato membro competente" ai sensi del regolamento n. 343/2003 quando non possono ignorare che le carenze sistemiche nella procedura di asilo e nelle condizioni di accoglienza dei richiedenti asilo in tale Stato membro costituiscono motivi seri e comprovati di credere che il richiedente corra un rischio reale di subire trattamenti inumani o degradanti ai sensi dell'art. 4 della Carta».

84. Corte europea dei diritti umani, Tarakhel c. Svizzera, cit., par. 104: «In the case of "Dublin" returns, the presumption that a Contracting State which is also the "receiving" country will comply with Article 3 of the Convention can therefore validly be rebutted where "substantial grounds have been shown for believing" that the person whose return is being ordered faces a "real risk" of being subjected to treatment contrary to that provision in the receiving country».

85. Corte suprema, Regno Unito, EM e altri, [2014] UKSC 12, sentenza del 19.2.2014, par. 58: «I consider that the Court of Appeal's conclusion that only systemic deficiencies in the listed country's asylum procedures and reception conditions will constitute a basis for resisting transfer to the listed country cannot be upheld [...]. The removal of a person from a member state of the Council of Europe to another country is forbidden if it is shown that there is a real risk that the person transferred will suffer treatment contrary to article 3 of ECHR».

86. In dottrina, taluni Autori hanno notato che l'attività ricognitiva dello Stato in ordine alla sussistenza del rischio sia la diretta conseguenza della configurazione dell'obbligo di non respingimento come obbligo di natura positiva. Cfr. in questo senso H. Battjes, In search of a fair balance: the absolute character of the prohibition of refoulement under article 3 ECHR reassessed, in Leiden Journal of International Law, 2009, pp. 601-603. Contra, nel senso di ritenere che l'obbligo sia solo negativo, si veda A. Gianelli, Obbligo di non refoulement e "assicurazioni diplomatiche", in Studi in onore di Vincenzo Starace, Napoli 2008, p. 281. Per un'analisi e un tentativo di sintesi dei due diversi orientamenti proposti in relazione alla giurisprudenza della Corte europea si veda ancora A. Tancredi, op. cit., pp. 47-50. 
Ed è chiaro, stante la delicatezza di tale ricognizione, che in alcun modo possano essere le autorità statali coinvolte in un'operazione di controllo alle frontiere esterne coordinata da Frontex a esaminare sulle imbarcazioni le richieste di protezione internazionale poiché sarebbe arduo garantire ad esse un'adeguata valutazione.

\section{Il ruolo di Frontex nelle operazioni di ricerca e salvataggio in mare: il dibattito in merito alla configurabilità di un "diritto a essere salvati"}

La tutela dei diritti umani nell'ambito di Frontex non può poi prescindere dal ruolo dell'Agenzia nelle operazioni di ricerca e salvataggio in mare.

La tragedia di Lampedusa dell'ottobre del 2013, come anticipato nelle premesse, ha imposto una riflessione sugli obblighi degli Stati in relazione a questo tema, che si è presto estesa al ruolo che l'UE, per il tramite di Frontex, deve ricoprire. ${ }^{87}$

Tale riflessione ha portato, di recente, all'istituzione, in primis e in ambito nazionale, dell'operazione Mare Nostrum, ora conclusa, che aveva l'obiettivo di soccorrere i migranti in situazioni di difficoltà. Mare Nostrum è stata poi sostituita da un'operazione coordinata da Frontex, Triton.

Le dichiarazioni dei vertici dell'Agenzia, relativamente a Triton, sono spesso criptiche circa le intenzioni di Frontex di effettuare operazioni di ricerca e soccorso, limitandosi a chiarire che il compito primario dell' Agenzia è quello di coordinare operazione di controllo alle frontiere. ${ }^{88}$

Ciononostante, almeno sulla carta, il tema della ricerca e del salvataggio in mare rientra nella nuova formulazione di cui al regolamento 656. La presenza di un articolo ad hoc - il numero 9 - introduce un elemento di riflessione di innegabile valore nell'economia delle operazioni di controllo alle frontiere esterne: sulla carta l'obbligo di prestare assistenza ai natanti rientra nell'acquis delle norme che vincolano l'Agenzia e gli Stati di volta in volta partecipanti alle operazioni. ${ }^{89}$

87. B. Nascimbene, L'Italia chiama..., cit., pp. 1394-1395.

88. In questo senso la dichiarazione del direttore esecutivo Gil Arias Fernandez del 31.10.2014, alla vigilia del lancio di Triton: «I would like to underline that operation Triton focuses on border control and surveillance. Having said that saving lives will remain an absolute priority for Frontex», disponibile sul sito internet frontex.europa.eu.

89. Regolamento (UE) n. 656/2014, cit., art. 9: «Gli Stati membri osservano l'obbligo di prestare assistenza a qualunque natante o persona in pericolo in mare e durante un'operazione marittima assicurano che le rispettive unità partecipanti si attengano a tale obbligo, conformemente al diritto internazionale e nel rispetto dei diritti fondamentali, indi- 


\section{A. Spagnolo}

Non solo. Anche il regolamento istitutivo di EUROSUR, che, come visto, ha l'obiettivo di rafforzare il coordinamento del controllo statale alle frontiere esterne sembra essere ispirato da una logica protettiva nei confronti della "salvezza dei migranti". 90

Il confortante quadro normativo appena descritto non fa però luce su un tema ancora oggetto di dibattito: i diritti e gli obblighi che derivano dal complesso quadro normativo della ricerca e salvataggio in mare. È prova di ciò il recente richiamo - operato dal direttore della divisione operativa di Frontex, Klaus Rosler, nei confronti del Governo italiano - con il quale l'Agenzia ha chiarito come una telefonata satellitare non possa sic et simpliciter attivare un'operazione di ricerca e soccorso «in zone poste fuori dall'area di operazione di Triton». ${ }^{91}$ Affermazioni di tale portata mettono in discussione l'efficacia delle operazioni coordinate da Frontex nel soccorso di migranti in difficoltà in alto mare.

Occorre quindi comprendere la portata dell'art. 9 del regolamento 656 in relazione alle norme di diritto internazionale che regolano le operazioni di ricerca e soccorso in mare.

Com'è noto, è nel diritto internazionale del mare che vanno ricercate, in primis, tali norme. L'art. 98 della Convenzione delle Nazioni Unite sul diritto del mare (CNUDM) impone agli Stati contraenti l'obbligo di imporre ai comandanti delle navi battenti la loro bandiera di prestare assistenza a natanti in difficoltà, ${ }^{92}$ e in questa direzione depone anche l'art. 10 della Convenzione sul salvataggio del $1989 .^{93}$

Sono poi le Convenzioni adottate nell'ambito dell'Organizzazione internazionale marittima (OIM), in particolare le Convenzioni SOLAS $^{94}$ e SAR, ${ }^{95}$ a imporre agli Stati un obbligo di cooperazione al fine di coordinare le rispettive competenze in relazione alle aree d'intervento.

pendentemente dalla cittadinanza o dalla situazione giuridica dell'interessato o dalle circostanze in cui si trova».

90. Regolamento (UE) n. 1052/2013, cit., considerando n. 1: «È necessario istituire un Sistema europeo di sorveglianza delle frontiere (EUROSUR) [...] al fine di $[\ldots]$ contribuire a garantire la protezione e la salvezza dei migranti».

91. La notizia è stata diffusa dall'Agenzia adnkronos il 9.12.2014 ed è reperibile sul sito internet www.adnkronos.com, con il titolo Immigrati, Frontex "preoccupata" per troppi interventi fuori area.

92. Convenzione delle Nazioni Unite sul diritto del mare, 10.12.1982, 1833 UNTS 397.

93. Convenzione internazionale sul salvataggio, 28.4.1989, 1953 UNTS 193.

94. Convenzione internazionale per la salvaguardia della vita umana in mare, 1.11.1974, 1184 UNTS 278, Capitolo V, Reg. 33.

95. Convenzione internazionale sulla ricerca e soccorso in mare, 27.4.1979, 1405 UNTS 97. 
Considerato di natura consuetudinaria,${ }^{96}$ l'obbligo giuridico che si ricava dalle predette norme è di mezzi e non di risultato. In altri termini, gli Stati non sono obbligati "a salvare" migranti in pericolo, bensì ad approntare tutte le misure necessarie per farlo e a vigilare che i comandanti delle navi che battono la loro bandiera agiscano nel rispetto dell' art. 98 CNUDM. ${ }^{97}$

Regolando rapporti interstatali, siffatti obblighi non contemplano, almeno $e$ xpressis verbis, un autonomo diritto "a essere salvati" in capo agli individui. ${ }^{98}$

È pur vero, però, che le stesse norme fanno riferimento a un "place of safety" nel quale devono essere sbarcati gli individui salvati in mare. Il riferimento al "luogo sicuro" contenuto nell'art. 3 (1) (9) della Convenzione SAR sembra implicare considerazioni relative alla tutela dei diritti umani, soprattutto di quei soggetti che, salvati in mare, desiderino fare domanda di protezione. Non è pensabile, quindi, che tali obblighi interstatali possano essere costruiti nel senso di esonerare gli Stati che operano il salvataggio dal rispetto dell'obbligo di non respingimento. ${ }^{99}$

Cionondimeno, proprio partendo dall'appena menzionato riferimento al "place of safety", vi è chi ritiene che le norme poc'anzi elencate possano essere interpretate nel senso di attribuire agli individui un diritto a essere salvati in mare ${ }^{100}$ conformemente a quell'orientamento dottrinale secondo cui il diritto internazionale del mare sarebbe da leggersi "human rights oriented". ${ }^{101}$

Il dibattito sul punto non è risolto, ma ciò non esclude che un "diritto a essere salvati" possa essere ricostruito a partire dagli obblighi derivanti

96. S. Trevisanut, Is there a right to be rescued at sea? A constructive view, in Questions of International Law, Zoom-in 4, 2014, p. 3 ss., in partic. p. 5.

97. E. Papastavridis, Is there a right to be rescued at sea? A skeptical view, in Questions of International Law, Zoom-in 4, 2014, p. 17 ss., in partic. p. 21.

98. Ibidem, p. 22. In questo senso, si può parlare di una sorta impermeabilità delle norme di diritto internazionale del mare rispetto a quelle in materia di diritti umani, cfr. Cfr. R. Barnes, The International Law of the Sea and Migration Control, in B. Ryan, V. Mitsilegas (eds.), op. cit., p. 112.

99. Valgano anche in queste sede le considerazioni svolte nel paragrafo precedente relativamente alla determinazione del luogo sicuro di sbarco. Cfr. supra, par. 4.

100. S. Trevisanut, Is there a right to be rescued at sea? A constructive view, cit., pp. 7-8. Contra, si veda ancora E. Papastavridis, op. ult. cit., p. 24.

101. S. Trevisanut, op. ult. cit., p. 7. Vi sono diversi Autori che affermano che le norme di diritto internazionale del mare vadano interpretate nell'ottica della tutela dei diritti umani. In questo senso si vedano, ex multis, T. Treves, Human Rights and the Law of the Sea, in Berkeley Journal of International Law, 2008, p. 1 ss.; I. Papanicolopulu, International Judges and the Protection of Human Rights at Sea, in International Courts and the Development of International Law, Essays in Honour of Tullio Treves, in N. Boschiero e altri (ed.), Amsterdam 2013, p. 535 ss. 


\section{A. Spagnolo}

dalle norme convenzionali in materia di diritti umani; in particolare, l'obbligo di prestare soccorso ai migranti in mare può essere derivato in via interpretativa dal diritto alla vita, sancito in numerosi Trattati internazionali.

La giurisprudenza, principalmente della Corte europea dei diritti umani, com'è noto, ha elaborato la dottrina degli obblighi positivi, che impone agli Stati membri della CEDU, entro certi limiti, l'obbligo di proteggere la vita degli individui posti sotto la propria giurisdizione ${ }^{102}$ in virtù di un'interpretazione evolutiva dell' art. 2 della Convenzione stessa. ${ }^{103}$

La costruzione di detti obblighi nei possibili scenari delle operazioni di Frontex va analizzata alla luce di quanto esposto nel paragrafo 3 del presente scritto: va, in primis, accertata l'applicazione extraterritoriale della norma pattizia che prevede l'obbligo.

Trattandosi di obblighi positivi, il controllo deve essere diffuso, o quantomeno essere tale da consentire allo Stato di intervenire. Ipotizzando uno scenario concreto, ${ }^{104}$ sembra lecito domandarsi se la "chiamata satellitare" che uno Stato dovesse ricevere da un natante in difficoltà, evocata dal direttore della sezione operativa di Frontex, sia sufficiente per estendere la giurisdizione di quello Stato sui migranti alla deriva.

Si può dare, contrariamente all'opinione di Klaus Rosler, una risposta positiva a condizione che lo Stato che riceve la chiamata sia a conoscenza delle esatte coordinate del luogo in cui si trova il natante, ${ }^{105}$ ciò che potrebbe, in ipotesi, soddisfare quel controllo diffuso necessario per estendere la giurisdizione statale.

La breve analisi appena proposta permette di trarre alcune conclusioni con riguardo alle operazioni coordinate da Frontex. Da un lato si può asse-

102. La dottrina degli obblighi positivi degli Stati in materia di diritti umani è trattata estensivamente in R. Pisillo Mazzeschi, Responsabilité de l'État pour violation des obligations positives relatives aux droits de l'homme, in Recueil des Cours de l'Académie de Droit International de La Haye, 2008, Leiden/Boston, 2009, pp.175-506.

103. F. Bestagno, Art. 2, in S. Bartole, P. De Sena, V. Zagrebelsky (a cura di), op. cit., p. 36 ss.

104. Uno dei casi senza dubbio più discussi è quello della c.d. "Left-to-die boat": un'imbarcazione di migranti partenti da Tripoli, in Libia, lasciata in balia delle acque nonostante, almeno così sembra, le autorità di alcuni Stati allora coinvolti nell'operazione della NATO Unified Protector avessero ricevuto - ed evidentemente ignorato - una chiamata di soccorso effettuata dallo stesso barcone. Sul punto, si rimanda alla risoluzione dell'Assemblea parlamentare del Consiglio d'Europa, Lives lost in the Mediterranean Sea: Who is responsible?, n. 1872(2012) reperibile sul sito internet assembly.coe.int.

105. S. Trevisanut, Is there a right to be rescued at sea? A constructive view, cit., p. 13 parla di «exclusive long distance de facto control». Conformemente si veda anche E. Papastavridis, op. ult. cit., p. 28-29. Anche quest'ultimo Autore parla esclusivamente di controllo de facto, escludendo che possa configurarsi un controllo de jure. 
rire che siano le norme in materia di diritto del mare a continuare a regolare gli aspetti di cooperazione legati alle operazioni di ricerca e soccorso in mare.

Ciononostante, è innegabile che la costruzione di un "diritto a essere salvati" nell'ambito delle norme sui diritti umani debba spingere i vertici operativi di Frontex a considerare la necessità di intervenire nel momento in cui, come visto, giunga una chiamata satellitare che identifichi, con precisione, le coordinate del natante in difficoltà, anche se tale luogo si collochi al di fuori delle aree d'intervento dell'operazione in questione.

Il mancato intervento in un siffatto scenario potrebbe, in ipotesi, essere oggetto di un ricorso in sede nazionale o internazionale (principalmente la Corte EDU) sulla responsabilità degli Stati partecipanti a un'operazione di Frontex - o l'Agenzia stessa - di prestare assistenza e soccorso.

\section{La responsabilità per violazioni dei diritti umani nell'ambito delle attività di Frontex}

Le considerazioni appena svolte circa l'esistenza - e l'azionabilità - di un diritto a essere salvati impongono l'introduzione di un tema cruciale nell'ambito delle operazione coordinate da Frontex: l'attribuzione delle eventuali condotte lesive dei diritti umani.

Nonostante Frontex abbia sempre escluso di potersi considerare responsabile per le condotte degli Stati membri partecipanti, ${ }^{106}$ una simile ipotesi non può, in principio, escludersi.

Le regole per inquadrare il tema della responsabilità nell'ambito di Frontex vanno ricercate sia negli Articoli sulla responsabilità degli Stati, sia in quelli sulla responsabilità delle organizzazioni internazionali, rispettivamente elaborati dalla Commissione del diritto internazionale delle Nazioni Unite e approvati, in seconda lettura, dall'Assemblea generale dell'ONU rispettivamente nel $2001^{107}$ e nel 2011. ${ }^{108}$

Preliminarmente, occorre dire che pochi dubbi ci devono essere circa l'attribuzione, delle condotte in violazione dei diritti umani poste in essere dagli Stati che partecipano alle operazioni di Frontex, agli Stati stessi. Le

106. Ciò che è stato affermato dall'Agenzia in occasione della prima indagine del Mediatore europeo: «As to the possibility of providing for a complaints mechanism for persons affected by its activities, Frontex pointed out that its task is "only to coordinate the cooperation of the EU Member States and Schengen Associated Countries"». Cfr. Decision of the European Ombudsmam As a consequence, only Member State authorities perform activities which may affect individuals' rights, cit., punto 22.

107. Commissione del diritto internazionale, UN Doc. A/56/10, p. 31.

108. Commissione del diritto internazionale, UN Doc. A/66/10, p. 55. 


\section{A. Spagnolo}

forze di polizia che materialmente prendono parte a una qualsiasi operazione sono e restano organi dello Stato d'invio; la loro condotta è, quindi, attribuibile allo Stato in virtù dell'art. 4 degli Articoli sulla responsabilità degli Stati.

Questo assioma può essere ribaltato ove si provasse che gli organi in questione siano posti sotto l'autorità di un altro soggetto: l'Agenzia stessa oppure lo Stato a cui è affidato il comando dell'operazione.

L'indagine può e deve tenere conto di diverse ipotesi, a seconda che a essere discussa sia la responsabilità dell'Agenzia, o quella degli Stati partecipanti alle singole operazioni.

La prima di esse potrebbe essere quella "classica" del "prestito di organi" da uno Stato a un' organizzazione internazionale, nel caso di specie l'UE.

Lo schema è stato individuato dalla Commissione del diritto internazionale nei lavori sulla codificazione responsabilità delle organizzazioni internazionali: gli Stati "prestano" alle organizzazioni internazionali propri organi per lo svolgimento di funzioni dell' organizzazione "ricevente".

Ai fini dell'attribuzione delle condotte, i rapporti che vengono a crearsi tra lo Stato e l'organizzazione internazionale, in un siffatto modello, sono descritti nell'art. 7 del Progetto del 2011. Questa norma attribuisce all'organizzazione internazionale "ricevente" le condotte dell' organo statale "prestato" a condizione che la prima abbia esercitato un "controllo effettivo" sulle seconde. ${ }^{109}$

È discutibile che quello appena esposto sia il modello che meglio descrive le operazioni di Frontex. L'art. 7, infatti, è pensato principalmente per le operazioni di peacekeeping delle Nazioni Unite, nell'ambito delle quali gli Stati mettono a disposizione dell'organizzazione propri contingenti militari trasferendo l'autorità e il comando su di essi alle Nazioni Unite. ${ }^{110}$

109. Sul punto si rimanda al secondo rapporto dello special rapporteur sulla responsabilità delle organizzazioni internazionali, G. Gaja, Second report on responsibility of international organizations, UN Doc., A.CN.4/541 del 2.4.2004, par. 31. Si veda anche dello stesso Autore, Responsabilité des Etats et/ou des organisations internationales en cas de violations des droits de l'homme, in La soumission des organisations internationales aux normes internationales relatives aux droits de l'homme, M. Forteau (ed.), Parigi 2009, p. 95 ss.

110. Cfr., di recente, sul tema, il dibattito pubblicato su Questions of International Law, Zoom-in 1, 2014, in partic. v. L. Condorelli, De la responsabilité internationale de l'ONU et/ou de l'État d'envoi lors d'actions de Forces de Maintien de la Paix: l'écheveau de l'attribution (double?) devant le juge néerlandais, p. 1 ss. e P. d'Argent, State organs placed at the disposal of the UN, effective control, wrongful abstention and dual attribution of conduct, p. 17 ss. 
Non sembra che vi sia un vero e proprio trasferimento d'autorità dagli Stati all'UE durante un'operazione Frontex: l'Agenzia, per sua natura, svolge un ruolo di mero coordinamento delle attività.

Analogamente sembra complessa da configurarsi ${ }^{111}$ l'ipotesi del "prestito d'organi" nel rapporto tra i diversi Stati partecipanti ad un'operazione di Frontex ai sensi dell'art. 6 degli articoli sulla Responsabilità degli Stati ${ }^{112}$ in relazione allo scenario in cui uno Stato assuma il comando di un'operazione a cui partecipino altri Stati e che le forze di polizia di questi ultimi siano poste sotto l'autorità del primo. ${ }^{113}$

Nei casi appena descritti, la condotta verrebbe attribuita unicamente a un soggetto: lo Stato o l'Agenzia a cui l'organo "viene prestato".

Maggiormente idonea a descrivere la complessità delle operazioni di Frontex, a cui partecipano diverse entità giuridiche, sembra essere la possibilità di attribuire simultaneamente una medesima condotta a più soggetti. ${ }^{1}$

Anche in questo caso occorre operare una distinzione sulla base del quadro normativo di riferimento.

Nell'ambito dei rapporti tra l'Agenzia e gli Stati membri che partecipano ad un'operazione sembra poteri configurare, in ipotesi, una responsabilità dell'UE per aver assistito gli Stati nella condotta lesiva ai sensi dell'art. 14 del Progetto di articoli sulla responsabilità delle organizzazioni internazionali. ${ }^{115}$

111. In questo senso si veda A. Liguori, N. Ricciuti, op. cit., pp. 555-556, per cui a escludere questa ipotesi sarebbe la sottoposizione delle guardie di frontiera ai poteri disciplinari dello Stato d'invio. Ancora, sempre in questa direzione, cfr. E. Papastavridis, "Fortress Europe" and Frontex..., cit., p. 107, per cui è difficile che si realizzi un controllo di intensità tale da ricondurre la condotta in questione allo Stato che "comanda" l'operazione.

112. « $[\mathrm{t}]$ he conduct of an organ placed at the disposal of a State by another State shall be considered an act of the former State under international law if the organ is acting in the exercise of elements of the governmental authority of the State at whose disposal it is placed» (corsivo aggiunto).

113. Regolamento (CE) n. 863/2007, cit., art. 5, co. 3: «I membri delle squadre possono svolgere compiti ed esercitare competenze esclusivamente agli ordini delle guardie di frontiera dello Stato membro ospitante e, di norma, in loro presenza». (corsivo nostro).

114. Come è stato sostenuto «If two states contribute to joint FRONTEX missions to control the external borders of the EU, and the rights of persons seeking asylum are violated, the question will arise whether the EU, and/or one or both of the states involved are responsible and, if so, how responsibility is distributed among them». Cfr. A. Nollkaemper, D. Jacobs, Shared Responsibility in International Law: a Conceptual Framework, in Michigan Journal of International Law, 2013.

115. Si veda ancora in questo senso A. Liguori, N. Ricciuti, op. cit., p. 556. L'art. 14 sulla responsabilità delle organizzazioni internazionali così recita: «An international organization which aids or assists a State or another international organization in the commission of an 


\section{A. Spagnolo}

Se l'indagine, invece, si sposta sul piano dei rapporti tra gli Stati partecipanti all'operazione le ipotesi sono diverse. ${ }^{116}$ La dottrina ha già evidenziato come l'art. 17 degli Articoli sulla responsabilità degli Stati possa essere una soluzione plausibile. ${ }^{117} \mathrm{Ai}$ sensi di quella norma è responsabile lo Stato che dirige o controlla un altro nella commissione di un illecito internazionale. ${ }^{118}$

Invero, i requisiti per l'applicazione dell' art. 17 sono piuttosto stringenti essendo limitata a «cases of domination over the commission of wrongful conduct and not simply the exercise of oversight, still less mere influence or concern». ${ }^{119}$ La configurazione di tale ipotesi, quindi, dipende dalla capacità dello Stato che assume il comando di un'operazione di Frontex di controllare la condotta di altri Stati partecipanti.

Uno scenario più attenuato è rappresentato dalla mera e più semplice circostanza in cui uno Stato aiuti o assista un altro nella commissione di un illecito, disciplinata dall'art. $16,{ }^{120}$ ciò che potrebbe verificarsi nel caso di azioni congiunte di Frontex con eventuali Stati terzi all'UE. ${ }^{121}$

internationally wrongful act by the State or the latter organization is internationally responsible for doing so if: (a) the former organization does so with knowledge of the circumstances of the internationally wrongful act; and (b) the act would be internationally wrongful if committed by that organization». Come si vedrà nel prossimo paragrafo, questa ipotesi, ancorché teoricamente solida, si scontra con le difficoltà, allo stato attuale, di convenire in giudizio l'UE, sia dinanzi alla Corte di giustizia, sia per la mancata, ad oggi, adesione dell'UE alla CEDU.

116. In generale sul punto v. M.L. Padelletti, Pluralità di stati nel fatto illecito internazionale, Milano 1990. Alla disciplina del tema è dedicato il capitolo IV degli Articoli sulla responsabilità degli Stati.

117. A. Liguori, N. Ricciuti, op. cit., p. 555.

118. «A State which directs and controls another State in the commission of an internationally wrongful act by the latter is internationally responsible for that act if: (a) that State does so with knowledge of the circumstances of the internationally wrongful act; and (b) the act would be internationally wrongful if committed by that State».

119. Yearbook of the International Law Commission, 2001, vol. II, Part Two, p. 69, par. 7.

120. «A State which aids or assists another State in the commission of an internationally wrongful act by the latter is internationally responsible for doing so if: (a) that State does so with knowledge of the circumstances of the internationally wrongful act; and (b) the act would be internationally wrongful if committed by that State».

121. Per una panoramica sul punto si rinvia a F. Cherubini, La cooperazione fra Unione europea e Paesi del Nordafrica nella lotta all'immigrazione irregolare, cit., pp. 37-39. 


\section{L'effettività dei diritti umani nell'ambito delle operazioni Frontex: un problema aperto}

La tutela dei diritti umani si fonda, necessariamente, sul principio di effettività. Ha senso parlare di applicabilità delle norme e di responsabilità solo se è consentito agli individui che lamentino una violazione dei diritti umani l'accesso a un sistema che dia loro la possibilità di ottenere ristoro.

Le operazioni congiunte coordinate da Frontex pongono problemi di non facile soluzione sotto questo profilo.

In particolare sembrano potersi isolare due temi di interesse: l'accesso a un rimedio giurisdizionale; il monitoraggio delle attività di Frontex.

L'accesso a un giudice è un diritto umano fondamentale, considerato ormai norma di natura consuetudinaria. Non vi sono pertanto ragioni di ritenere che esso non si debba configurare nell'ambito delle operazioni coordinate da Frontex.

Ciò che complica la realizzazione di un siffatto diritto è indubbiamente l'inesistenza di un sistema di ricorsi interno all'Agenzia che consenta all'UE di gestire eventuali reclami. Tale situazione è la logica conseguenza della posizione, netta, di Frontex con riguardo all'esclusiva responsabilità degli Stati partecipanti alle varie operazioni in mare. Non può stupire che un'Agenzia che non rivendica per sé le condotte dei soggetti che partecipano alle proprie operazioni, non abbia istituito un sistema di gestione dei ricorsi per violazioni dei diritti umani.

Di dubbia efficacia è anche l'esistenza di una competenza, del tutto teorica, della Corte di giustizia dell'UE a sindacare gli atti dell'Agenzia. ${ }^{122}$ Non può però essere esclusa questa competenza in merito a richieste di risarcimento per danni da responsabilità extracontrattuale, prevista nel regolamento istitutivo di Frontex, il 2007/2004, nell'art. $19 .{ }^{123}$ Com'è stato notato, tale competenza non pare essere del tutto efficace, rimanendo vincolata a un effettivo monitoraggio delle attività poste in essere nell'ambito di Frontex, ciò che, come vedremo più avanti, è un aspetto problematico della gestione dell' Agenzia. ${ }^{124}$

122. È stato notato come tale competenza sia solo teorica, risultando difficile che gli atti dell'Agenzia, anche a seguito delle modifiche introdotte dal Trattato di Lisbona, possano essere produttivi di effettivi nei confronti di terzi, che è la condizione al verificarsi della quale gli atti di organi e organismi dell'UE possono essere sottoposti a un giudizio di sindacato da parte della Corte di giustizia dell'UE. Cfr. F. Cherubini, op. cit., pp. 35-36.

123. Regolamento (CE) n. 2007/2004, cit., art. 19.

124. In questo senso ancora F. Cherubini, La cooperazione fra Unione europea e Paesi del Nordafrica nella lotta all'immigrazione irregolare, cit., p. 36; si vedano anche A. Fischer-Lescano, T. Löhr, T. Tohidipur, op. cit., p. 295. 


\section{A. Spagnolo}

In un siffatto scenario le possibilità per un individuo di ottenere ristoro si riducono essenzialmente all'esperimento di quelle vie di ricorso che prevedano la proposizione di istanze contro gli Stati partecipanti alle operazioni di Frontex.

Qui va necessariamente riconosciuto un ruolo alle giurisdizioni degli Stati nazionali e a quella della Corte EDU.

Le due strade sono solo all'apparenza semplici da percorrere. La proposizione di ricorsi in sede nazionale presuppone, infatti, che le Corti interne si dichiarino competenti ratione personae a giudicare la condotta degli organi statali inviati nell'ambito dell'operazione in questione. In una siffatta prospettiva, il rischio è quello che le Corti interne possano dichiararsi incompetenti a sindacare atti che, formalmente, dovrebbero attribuirsi all'UE, oppure allo Stato a cui è affidato il comando dell'operazione, nell'ipotesi in cui non sia quello convenuto. Abbiamo già notato come tale impostazione non possa reggere, ma non può del tutto escludersi, soprattutto in quelle operazioni in cui i partecipanti appartengono a Stati diversi.

Parimenti, ma per altri motivi, anche la proposizione di un ricorso alla Corte EDU non appare scontata. A essere problematico, qui, oltre al tema della competenza ratione personae della Corte, è il tema dell'applicazione extraterritoriale della CEDU.

Inevitabilmente, arrivati a questo punto dell'indagine, tutti gli spunti fin qui trattati concorrono a delineare un quadro di incertezza circa gli strumenti a disposizione di eventuali vittime di violazioni dei diritti umani patite nell'ambito dell'attività di Frontex.

Incertezza che poteva essere dissipata - o quantomeno limitata dall'adesione dell'UE alla CEDU, prevista nell'art. 6 co. 3 del TUE e già definita, in sede negoziale, in una bozza di accordo tra le due istituzioni.

Diversi commentatori indicavano questa prospettiva come risolutiva rispetto a scenari simili a quello appena descritto, se non addirittura in termini, ${ }^{126}$ soprattutto in ordine alla possibilità, prevista nell'accordo di adesione che l'UE potesse essere chiamata in causa come co-respondent in procedimenti contro gli Stati membri per atti che questi ultimi avessero posto in essere, ${ }^{127}$ concretizzando, quindi, le ipotesi di responsabilità descritte nel paragrafo precedente.

125. Vedasi Fifth negotiation meeting between the CDDH Ad Hoc Negotiation Group and the European Commission on the accession of the European Union to the European Convention on Human Rights, 47+1(2013)008rev2, 5.4.2013, reperibile sul sito internet del Consiglio d'Europa: www.coe.int.

126. Ancora cfr. F. Cherubini, op. cit., p. 42; A. Liguori, N. Ricciuti, op. cit., p. 566.

127. S. Vezzani, The UE and Its Member States before the Strasbourg Court: a Critical Appraisal of the Co-respondent Mechanism, in The Constitutional Relevance of the ECHR 
Il parere negativo della Corte di giustizia sull'adesione dell'UE alla CE$\mathrm{DU}^{128}$ ha bloccato, per ora, lo sviluppo delle ipotesi sopra menzionate. La netta presa di posizione della Corte, destinata a far parlare di sé, ${ }^{129}$ chiude alla possibilità che giurisdizioni esterne all'UE siano competenti a sindacare atti dell'Unione e che l'UE stessa possa essere parte di un giudizio dinanzi alla Corte di Strasburgo. Inevitabilmente, il parere 2/13 della Corte di giustizia chiude anche alla possibilità che la responsabilità di Frontex possa essere discussa dinanzi alla Corte EDU.

Rimangono in piedi le soluzioni che comportano la proposizione di ricorsi contro gli Stati partecipanti alle operazioni coordinate da Frontex, limitatamente alla loro esclusiva responsabilità.

Non è detto, però, che le soluzioni giurisdizionali rappresentino gli unici strumenti di garanzia di tutela dei diritti umani.

\section{Segue. Il monitoraggio dei diritti umani nell'ambito di Frontex: una possibile conclusione}

Stante lo scenario appena descritto - non certo ottimistico in relazione alle vie di ricorso giurisdizionali disponibili alle vittime di violazioni dei diritti umani - è necessario immaginare altre modalità per garantire un'effettiva tutela dei diritti durante le operazioni di controllo alle frontiere esterne.

È il monitoraggio delle operazioni di Frontex, infatti, a dover essere rafforzato. In questa prospettiva, non può che essere salutata con favore l'istituzione di un responsabile dei diritti fondamentali nell'ambito dell'Agenzia, ciò che pare, effettivamente, rendere centrale il ruolo di

in Domestic and European Law. An Italian Perspective, G. Repetto (a cura di), Anversa 2012, p. 32 ss.

128. Com'è noto, l'adesione dell'UE alla CEDU, prevista nell'art. 6 del TUE, era subordinata al parere positivo della Corte di giustizia dell'UE ex art. 218 TFUE. Il suddetto parere è stato reso dalla Corte di Lussemburgo il 18.12.2014 su richiesta della Commissione e previa presa di posizione, anch'essa negativa, dell'avv. generale Julianne Kokott. V. Richiesta di parere presentata dalla Commissione europea ai sensi dell'art. 218, paragrafo 11, TFUE (Parere 2/13) (2013/C 260/32), in GUUE C 260 del 7.9.2013, p. 19.

129. Per ora si segnalano solo commenti reperibili on line, tra i quali vedansi: L.S. Rossi, Il Parere 2/13 della CGUE sull'adesione dell'UE alla CEDU: scontro fra Corti? e S. Vezzani, "Gl'è tutto sbagliato, gl'è tutto da rifare!": la Corte di giustizia frena l'adesione dell'UE alla CEDU, entrambi pubblicati sul blog della Società italiana di diritto internazionale (SIDIBlog), www.sidi-isil.org/sidiblog; vedasi anche I. Anrò, Il parere 2/13 della Corte di giustizia sul progetto di accordo di adesione dell'Unione europea alla CEDU: una bocciatura senza appello?, su www.eurojus.it. 


\section{A. Spagnolo}

Frontex nel rispetto dei diritti a un piano operativo. ${ }^{130} \mathrm{E}$, peraltro, in questo quadro e con queste premesse che può essere inserita l'adozione del regolamento istitutivo di EUROSUR, che ha l'obiettivo di rafforzare il coordinamento del controllo statale alle frontiere esterne. Com'è stato notato, ${ }^{131}$ l'istituzione di EUROSUR non ha solo rilevanza ai fini della lotta alla criminalità organizzata, ma anche, e oseremmo dire soprattutto, in relazione alla tutela dei diritti fondamentali. ${ }^{132}$

Le suddette, importanti, innovazioni legislative devono essere accompagnate, necessariamente, da un monitoraggio effettivo delle operazioni coordinate da Frontex, ciò che sembra essere il vero punctum dolens dell'attività dell' Agenzia.

Non è un caso, quindi, che il 20 ottobre scorso, il Mediatore europeo (European Ombudsman), Ms. Emily O'Reilly abbia avviato, proprio motu, un'indagine sull'attività dell'Agenzia in relazione alle cosiddette Joint Return Operations (operazioni di rimpatrio). ${ }^{133}$ L'attività legata ai rimpatri è centrale nell'azione di Frontex: stando agli ultimi dati trasmessi dalla Commissione europea, dal 2006 al 2013 l'Agenzia ha coordinato 209 ope-

130. B. Nascimbene, Lampedusa: una tragedia con molti interrogativi..., cit., p. 9, parla di un quadro "prevenzione e controllo" destinato a prevalere.

131. B. Nascimbene, op. ult. cit., p. 10.

132. Regolamento (UE) n. 1052/2013, cit., considerando n. 11 («Il presente regolamento rispetta i diritti fondamentali e osserva i principi riconosciuti dagli artt. 2 e 6 del Trattato sull'Unione europea (TUE) e dalla Carta dei diritti fondamentali dell'Unione europea, in particolare il rispetto per la dignità umana, il diritto alla vita, la proibizione della tortura e di trattamenti o pene inumani o degradanti, la proibizione della tratta di esseri umani, il diritto alla libertà e alla sicurezza, il diritto alla protezione dei dati personali, il diritto di accesso ai documenti, il diritto di asilo, la protezione contro l'allontanamento e l'espulsione, il divieto di respingimento, il divieto di discriminazione e i diritti del minore. Gli Stati membri e l'Agenzia dovrebbero applicare il presente regolamento in conformità di tali diritti e principi».) e 12 («Ai sensi del regolamento (CE) n. 2007/2004, il responsabile dei diritti fondamentali e il forum consultivo istituito dallo stesso regolamento dovrebbero avere accesso a tutte le informazioni riguardanti il rispetto dei diritti fondamentali in relazione a tutte le attività dell'Agenzia nella struttura di EUROSUR»). Peraltro, si richiama il già citato considerando $n$. 1 del regolamento istitutivo di EUROSUR, che fa riferimento alla necessità di proteggere la vita dei migranti.

133. Si veda European Ombudsman, Own initiative inquiry OI/9/2014/MHZ concerning the means through which Frontex ensures respect for fundamental rights in joint return operations (JRO). 
Interventi

razioni, rimpatriando circa 11.000 persone. ${ }^{134}$ Solo la metà di dette operazioni è stata efficacemente monitorata. ${ }^{13}$

Non sono ancora disponibili le risposte dell'Agenzia alle sollecitazioni del Mediatore europeo; è chiaro, però, che la quasi totale assenza di un sistema di rimedi giurisdizionali impone alla direzione di Frontex un'attenzione particolare verso l'istituzione di meccanismi di monitoraggio efficaci che possano garantire che i diritti fondamentali dei migranti siano tutelati.

134. Si veda Communication from the Commission to the Council and the European Parliament on EU Return Policy, COM(2014) 199 final, 28.3.2014.

135. Ibidem, par. 4.2, pp. 5-6. 